\title{
Editorial: I have an avatar therefore I exist
}

\author{
Savvas Papagiannidis • Michael Bourlakis • Feng Li
}

Published online: 10 March 2009

(C) Springer Science+Business Media, LLC 2009

This special issue explores the rapid development of massively multiplayer online role playing games (MMORPG) and contributes to our understanding of this fast growing research area, with particular emphasis on their business and social implications. This is reflected in the research questions addressed by the papers published in this special issue.

Alex Meredith, Zaheer Hussain and Mark Griffiths undertook a scoping study to examine the extent, range and nature of different MMORPGs. Their research aims were first to present a summarised overview of all the material reviewed and secondly to provide a thematic construction in order to present a narrative account of existing MMORPG literature. Their findings suggest that whilst some games have received moderate or even substantial attention, others have had no research conducted upon them at all. This presents a problem, since the growth in both the player base and the industry suggests that a single psychological profile of 'the gamer' cannot be relied upon.

Kim Bryceson, on the other hand, has looked at the development of an animated, interactive, three-dimensional virtual environment model. More specifically, she presented VAG - a prototype of such a virtual environment developed as a CD-based game of strategy in the context of a supply chain in the agribusiness sector. Apart from the development of the first version and a qualitative evaluation of the demonstration model she discusses the future developmental opportunities for extending VAG into a massive multiplayer online game in true virtual reality.

S. Papagiannidis $(\varangle) \cdot$ F. Li

Newcastle University, Newcastle, UK

e-mail: savvas.papagiannidis@ncl.ac.uk

M. Bourlakis

Brunel University, London, UK 
Maria Rosita Cagnina and Michele Poian also conducted a study on a virtual world level. They have qualitatively examined how owning and maintaining a virtual world can impact on the business models of firms, by identifying value drivers and the subsequent impact on elements of the value propositions. Their findings could offer support to firms looking to virtual worlds as a new way to implement a winning business model.

The next two papers offer evidence related to users' purchasing behaviour in virtual worlds. Yue Guo and Stuart Barnes aimed to contribute to the relevant literature by identifying the factors that influence such behaviour using a focus group approach. Their preliminary findings suggest that virtual item purchasing behaviour has considerable variability, involving a range of factors at different stages.

Vili Lehdonvirta has studied purchases in virtual worlds from a different angle, that of real money trading. Selling virtual goods for real money is an increasingly common revenue model, not only for online games and virtual worlds, but also for social networking sites and other mainstream online services. Lehdonvirta adopted a perspective from the sociology of consumption, analysing examples from 14 virtual asset platforms to suggest a more detailed set of item attributes that drive virtual item purchase decisions, consisting of functional, hedonic and social attributes.

Lakshmi Goel and Sonja Prokopec examine the differences between virtual worlds and traditional virtual channels such as websites, and suggest effective entry strategies for organisations that seek to leverage their presence in virtual worlds. The results of their qualitative and quantitative analysis indicate that there is a significant difference in perceptions of trust, product diagnosticity, informativeness, and product descriptions between websites and virtual worlds. The paper's results also suggest that organisations need to employ a synergy strategy when marketing experience goods in virtual worlds.

Finally, Michael Bourlakis, Savvas Papagiannidis and Feng Li have examined the evolution of retailing, i.e. the transition from traditional to electronic to metaverse retailing, taking into consideration the spatial dimension in the context of the unique characteristics of one virtual world, that of Second Life. Their key finding is that retailers need to employ a holistic and overarching approach when devising their promotional strategies, especially if they aim to operate at the metaverse stage as well.

These papers provide new insights into virtual worlds and at the same time pose even more questions that need to be addressed by further research. We hope you find this special issue informative and challenging, and welcome your constructive criticisms and comments. 